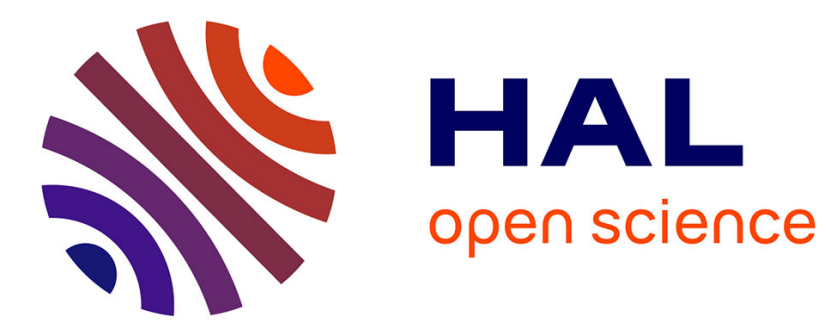

\title{
EasySDM: An Integrated and Easy to Use Spatial Data Mining Platform
}

\author{
Leila Hamdad, Amine Abdaoui, Nabila Belattar, Mohamed Ala
}

\section{To cite this version:}

Leila Hamdad, Amine Abdaoui, Nabila Belattar, Mohamed Ala. EasySDM: An Integrated and Easy to Use Spatial Data Mining Platform. KDIR: Knowledge Discovery and Information Retrieval, Nov 2015, Lisbonne, Portugal. lirmm-01229030

\section{HAL Id: lirmm-01229030 https://hal-lirmm.ccsd.cnrs.fr/lirmm-01229030}

Submitted on 16 Nov 2015

HAL is a multi-disciplinary open access archive for the deposit and dissemination of scientific research documents, whether they are published or not. The documents may come from teaching and research institutions in France or abroad, or from public or private research centers.
L'archive ouverte pluridisciplinaire HAL, est destinée au dépôt et à la diffusion de documents scientifiques de niveau recherche, publiés ou non, émanant des établissements d'enseignement et de recherche français ou étrangers, des laboratoires publics ou privés. 


\title{
EasySDM: An Integrated and Easy to Use Spatial Data Mining Platform
}

\author{
Leila Hamdad $^{1}$, Amine Abdaoui ${ }^{2}$, Nabila Belattar ${ }^{1}$, and Mohamed Ala Alchikha ${ }^{1}$ \\ ${ }^{l}$ LSCI, ESI, BP $68 M$ Oued Smar, Algiers, Algeria \\ ${ }^{2}$ LIRMM, 860 St Priest Street, Montpellier, France \\ $\left\{l \_h a m d a d, n \_b e l a t t a r, m \_a l \_c h i k h a\right\} @ e s i . d z, a b d a o u i @ l i r m m . f r$
}

Keywords: $\quad$ Spatial Data Mining, Geo-visualization, Classification, Clustering, Association Rules.

\begin{abstract}
Spatial Data Mining allows users to extract implicit but valuable knowledge from spatial related data. Two main approaches have been used in the literature. The first one applies simple Data Mining algorithms after a spatial pre-processing step. While the second one consists of developing specific algorithms that considers the spatial relations inside the mining process. In this work, we first present a study of existing Spatial Data Mining tools according to the implemented tasks and specific characteristics. Then, we illustrate a new open source Spatial Data Mining platform (EasySDM) that integrates both approaches (pre-processing and dynamic mining). It proposes a set of algorithms belonging to clustering, classification and association rule mining tasks. Moreover and more importantly, it allows geographic visualization of both the data and the results. Either via an internal map display or using any external Geographic Information System.
\end{abstract}

\section{INTRODUCTION}

Spatially related data is present in many fields such as epidemiology, environmental science, image analysis, etc. In fact, many problems are spatially dependent. The study of any characteristic of such data can not be done without taking into account their respective geographical positions. In its most common form, a spatial data is divided into two parts: a descriptive part that can be of any standard type (Integer, Boolean, etc.) and a geographic part describing the geometry and geo-spatial position of the data. Voluminous geographic data are being collected with modern acquisition techniques such as Global Positioning Systems (GPS), highresolution remote sensing, Geographic Information System (GIS), etc. Extracting unknown and unexpected information from these spatial data sets requires efficient methods that take into account the spatial dependencies (Guo, 2009). Spatial data are characterized by their interdependence, which comes from the following assumption: "The more objects are close to each other, the higher is the correlation between them" (Miller, 2004). Therefore, Spatial Data Mining (SDM) has emerged as an active area of research for extracting implicit and relevant knowledge from large spatial databases containing great, complex and interdependent data (Anselin et al., 2006). In general, SDM tasks are extensions of Data Mining (DM) ones by taking into account spatial relations. These tasks include clustering, classification, association rule mining and geovisualization.

In the literature, several software of SDM exist, which function according to two main approaches. The first approach, which is the most intuitive one, consists of using classical DM algorithms on preprocessed spatial data. The pre-processing consists of extracting smoothed data table from the matrix between neighbouring objects, or by representing the spatial relations as new features (Ouattara, 2010; Rinzivillo et al, 2008). This approach is simple but time consuming (Guo, 2008). The second approach consists of developing specific SDM techniques that dynamically takes into consideration spatial relationships inside the mining process. Therefore, the exploratory process of this approach is faster than the first one but more complicated to implement.

In this work, we first propose a study on existing SDM tools focusing on their proposed tasks and specific characteristics. To our knowledge, no similar study has been proposed before in order to compare SDM tools, in contrast to the huge work done to compare classical DM tools (Goebel and Gruenwald, 1999; John F. Elder and Dean W. Abbott, 1998; Witten and Frank, 2005). Then, we 
present EasySDM, our new integrated, open source and easy to use SDM platform. It integrates algorithms from both pre-processing and dynamic SDM approaches. On the one hand, algorithms from the Weka DM tool (Hall et al., 2009) have been used after a pre-processing step using the GDPM API (Bogorny et al., 2006). On the other hand, a naïve regionalization algorithm and a simple spatial rules association extraction algorithm that can be directly applied on spatial data have been implemented. While existing SDM tools show a lack of visualisation especially for open source ones, EasySDM offers the possibility to visualize spatial data directly on an integrated geographical map before and after applying DM algorithms. Furthermore, a visualization is also possible via any external Geographic Information System (GIS). Due to its simplicity and visualization capabilities, we believe that EasySDM may be helpful, inter alia, in explaining SDM to students in the academic area. It has been produced under the GPL licence in order to allow researchers and programmers to access and improve the source code. The platform setup, source code and documentation are publically available on the internet ${ }^{1}$.

The rest of the paper is organized as follow: First, a comparative study on existing SDM tools is presented in section 2. Then, EasySDM and its components are detailed in section 3. After that, we conduct some experiments using EasySDM in order to illustrate its functionalities and present them in section 4. Finally, section 5 concludes and gives our main perspectives.

\section{COMPARATIVE STUDY OF SDM TOOLS}

Many SDM tools have been proposed in the literature. (Han et al., 1997) proposed GeoMiner, the first knowledge extraction software from spatial databases, developed in 1997. It is an extension of the classical DM tool DBMiner (Jiawei Han, 1996) developed by the same team in 1996. Similarly, (Ouattara, 2010) developed GeoKnime, an extension of the Knime software (www.knime.org) to spatial data. (Appice et al., 2007) proposed Ingens, an integrated platform for SDM within a GIS environment. (Lazarevic et al., 2000) developed SDAM, a software system for spatial data analysis and modelling that includes two tasks of SDM (clustering and classification). (May and Savinov, 2001) developed the SPIN system, a spatial information system that implements many clustering, classification and association rule mining algorithms. (Bogorny et al., 2006) developed a spatial pre-processing API that can be added to the Weka software in order to treat spatial data. Finally, an interesting application of clustering, named CrimeStat, has been proposed in (Levine and al, 2004) in order to detect hot spots of crime incidents.

In this section, we compare these tools according to their general characteristics. Table 1 presents for each tool: the year of its latest release, whether the software and the source code are publically accessible or not, whether a documentation is available or not and, finally, the type of the proposed visualization (if any).

Table 1 : General characteristics of existing SDM tools

\begin{tabular}{|c|c|c|c|c|c|c|}
\hline $\begin{array}{l}\stackrel{\Xi}{\Xi} \\
\stackrel{\Xi}{\Xi} \\
\bar{O} \\
\stackrel{\circ}{\ominus}\end{array}$ & 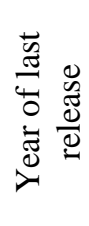 & 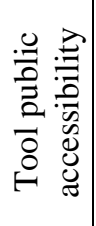 & 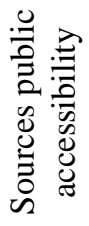 & 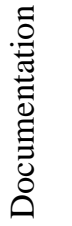 & 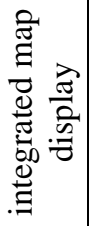 & 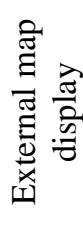 \\
\hline $\begin{array}{l}\text { Geo- } \\
\text { Miner }\end{array}$ & 1999 & No & No & No & Yes & No \\
\hline $\begin{array}{l}\text { Geo- } \\
\text { Knime }\end{array}$ & 2010 & No & No & No & No & No \\
\hline Ingens & 2007 & No & No & No & Yes & No \\
\hline SDAM & 2000 & No & No & No & No & No \\
\hline SPIN & 2003 & Yes & No & Yes & Yes & No \\
\hline GDPM & 2007 & Yes & Yes & Yes & No & No \\
\hline $\begin{array}{l}\text { Crime- } \\
\text { Stat }\end{array}$ & 2010 & Yes & No & Yes & Yes & Yes \\
\hline
\end{tabular}

Table 2 presents a comparison of these tools according to their technical characteristics. For each tool, it presents its architecture, the programming language, whether it functions with all operating systems, and the possible types of data input. Finally, Table 3 presents a functional comparison, which takes into consideration the used SDM approach, the types of the considered spatial relations, and the implemented SDM tasks.

\footnotetext{
${ }^{1}$ http://www.lirmm.fr/ abdaoui/EasySDM
} 
Table 2: Technical characteristics of SDM tools (Un: Unknown, Win: Windows)

\begin{tabular}{|c|c|c|c|c|}
\hline $\begin{array}{l}\stackrel{\Xi}{\Xi} \\
\stackrel{\Xi}{\Xi} \\
\stackrel{0}{0} \\
\end{array}$ & 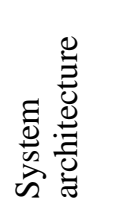 & 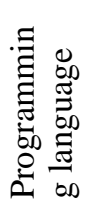 & 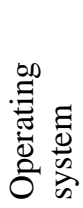 & 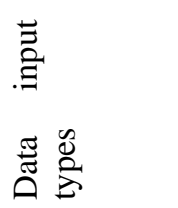 \\
\hline GeoMiner & $\begin{array}{l}\text { Client/ } \\
\text { Server }\end{array}$ & Un & All & Database \\
\hline GeoKnime & Desktop & Java & All & Database \\
\hline Ingens & $\begin{array}{l}\text { Client/ } \\
\text { Server }\end{array}$ & Java & All & Database \\
\hline SDAM & Desktop & $\mathrm{C}++$ & Win & Database \\
\hline SPIN & N-tier & Java & All & Database \\
\hline GDPM & Desktop & Java & All & Database \\
\hline CrimeStat & Desktop & $\mathrm{C}++$ & All & $\begin{array}{l}\text { Files: dbf, } \\
\text { shp and dat }\end{array}$ \\
\hline
\end{tabular}

Table 3: SDM characteristics of SDM tools (Pre-pro: Preprocessing, D: Distance, T: Topological, R: Directional, Un: Unknown)

\begin{tabular}{|c|c|c|c|c|c|}
\hline \multirow[t]{2}{*}{ Tool name } & \multirow{2}{*}{$\begin{array}{l}\text { SDM } \\
\text { approach }\end{array}$} & \multirow{2}{*}{$\begin{array}{l}\text { Spatial } \\
\text { relations }\end{array}$} & \multicolumn{3}{|c|}{ SDM tasks } \\
\hline & & & 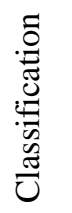 & $\begin{array}{l}\stackrel{00}{\Xi} \\
\stackrel{\Xi}{0} \\
\stackrel{0}{\Xi} \\
\stackrel{\Xi}{\Xi}\end{array}$ & 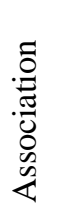 \\
\hline GeoMiner & Dynamic & $\mathrm{D}$ & Yes & Yes & Yes \\
\hline GeoKnime & Dynamic & $\mathrm{D}$ and $\mathrm{T}$ & Yes & Yes & No \\
\hline Ingens & Dynamic & $\begin{array}{l}\mathrm{D}, \mathrm{T} \text { and } \\
\mathrm{R}\end{array}$ & No & No & Yes \\
\hline SDAM & Pre-pro & Un & Yes & Yes & Yes \\
\hline SPIN & Dynamic & $\mathrm{D}$ and $\mathrm{T}$ & Yes & Yes & Yes \\
\hline GDPM & Pre-pro & $\mathrm{D}$ and $\mathrm{T}$ & Yes & Yes & Yes \\
\hline CrimeStat & Pre-pro & $\mathrm{D}$ and $\mathrm{T}$ & No & No & Yes \\
\hline
\end{tabular}

It is important to notice that GeoMiner and Ingens have been built on specific spatial query languages. When they were released, these two tools were not successful. Moreover, GeoKnime and SDAM are not publically accessible and do not seem to be massively used. Since we could not test these four tools, their characteristics have been extracted from the scientific papers describing them.

\section{SYSTEM OVERVIEW}

\subsection{Architecture}

As presented in the previous section, Weka- GDPM is accessible, open source and includes the main three SDM tasks using the pre-processing approach. However, it does not provide any geographical visualization of the results. Therefore, we decided to use and enrich Weka-GDPM with a geographic visualization. Figure 1 below presents the platform architecture. The visualization of the spatial data and the results can be performed within the platform or using any external GIS. The internal map display has been implemented using the MapWinGIS API. The external visualization can be done by any GIS to open the data and the results. In addition to the Weka algorithms that can be applied on preprocessed data, we implemented a naïve regionalization and a simple spatial rule association mining algorithms (spatial Apriori) that can be applied directly on spatial data without any preprocessing. The development was carried out on a Microsoft Visual Studio 2012 platform using the C\# programming language. The jar files of Weka and GDPM have been converted to dll files accessible from the $\mathrm{C \#}$ code using the IKVM tool (www.ikvm.net). The data sources can be either arff files (.arff), shape files (.shp) or a PostGis 2.0 database. The obtained results can be saved in .arff or .shp formats.

\subsection{Graphical User Interface}

The Graphical User Interface (GUI) is simple and intuitive, hence the name of EasySDM. As presented in Figure 2, the GUI is divided into three areas:

- Region A (red rectangle): Allows the user to interact with the platform by specifying the shape file and the .arff file, setting up the parameters, modifying the theme of or the attribute to be displayed on the map, lunching the algorithm, and saving the results.

- Region B (green rectangle): Displays information about the run such as: the algorithm status, the success/fail of the algorithm, the execution time and error messages, etc.

- Region C (blue rectangle): This area is dedicated to the map display and the legend. It allows to visualize on the map both the data and the results. For example the same colour is used for objects that are in the same cluster. 


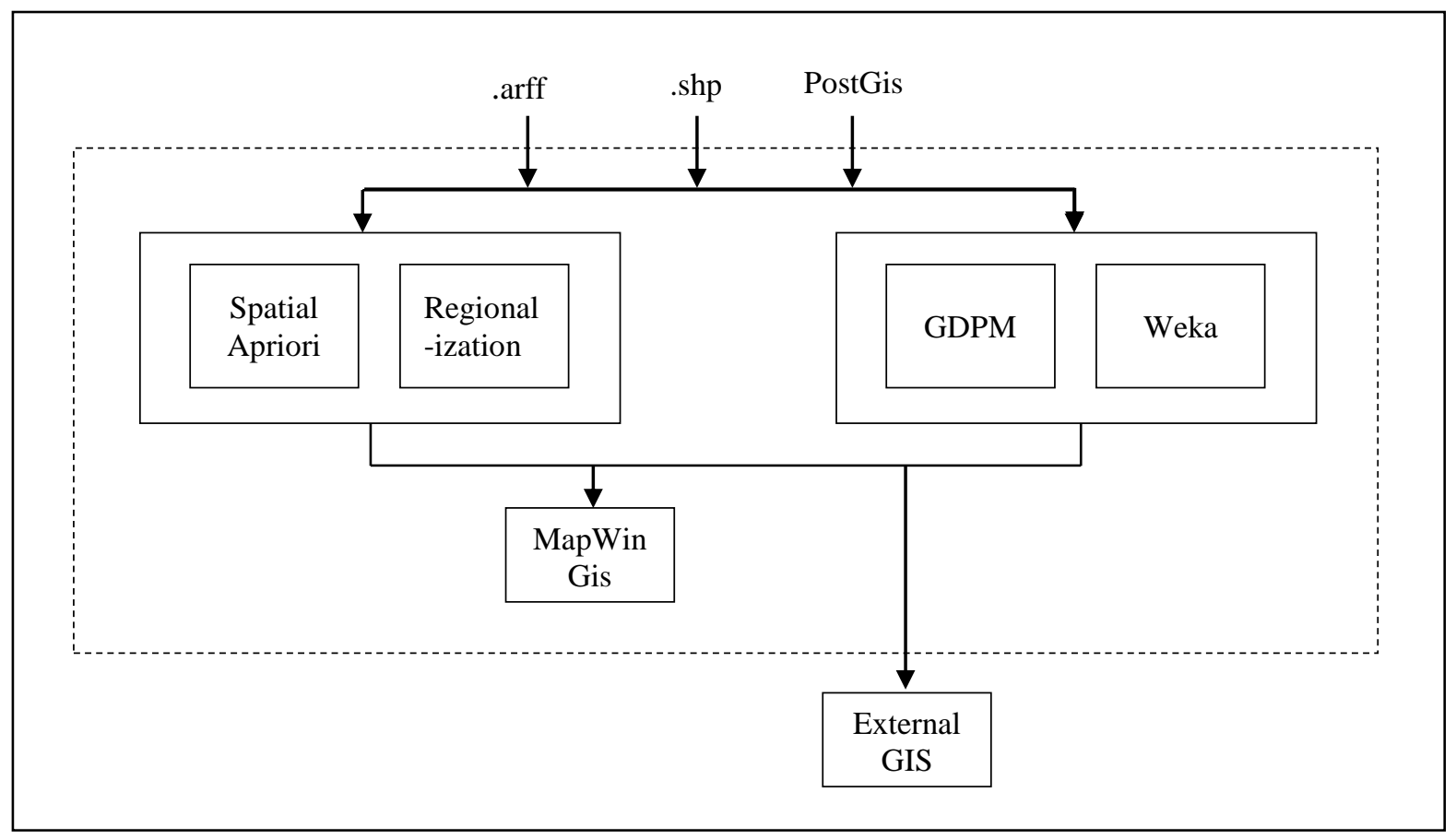

Figure 1: EasySDM architecture.

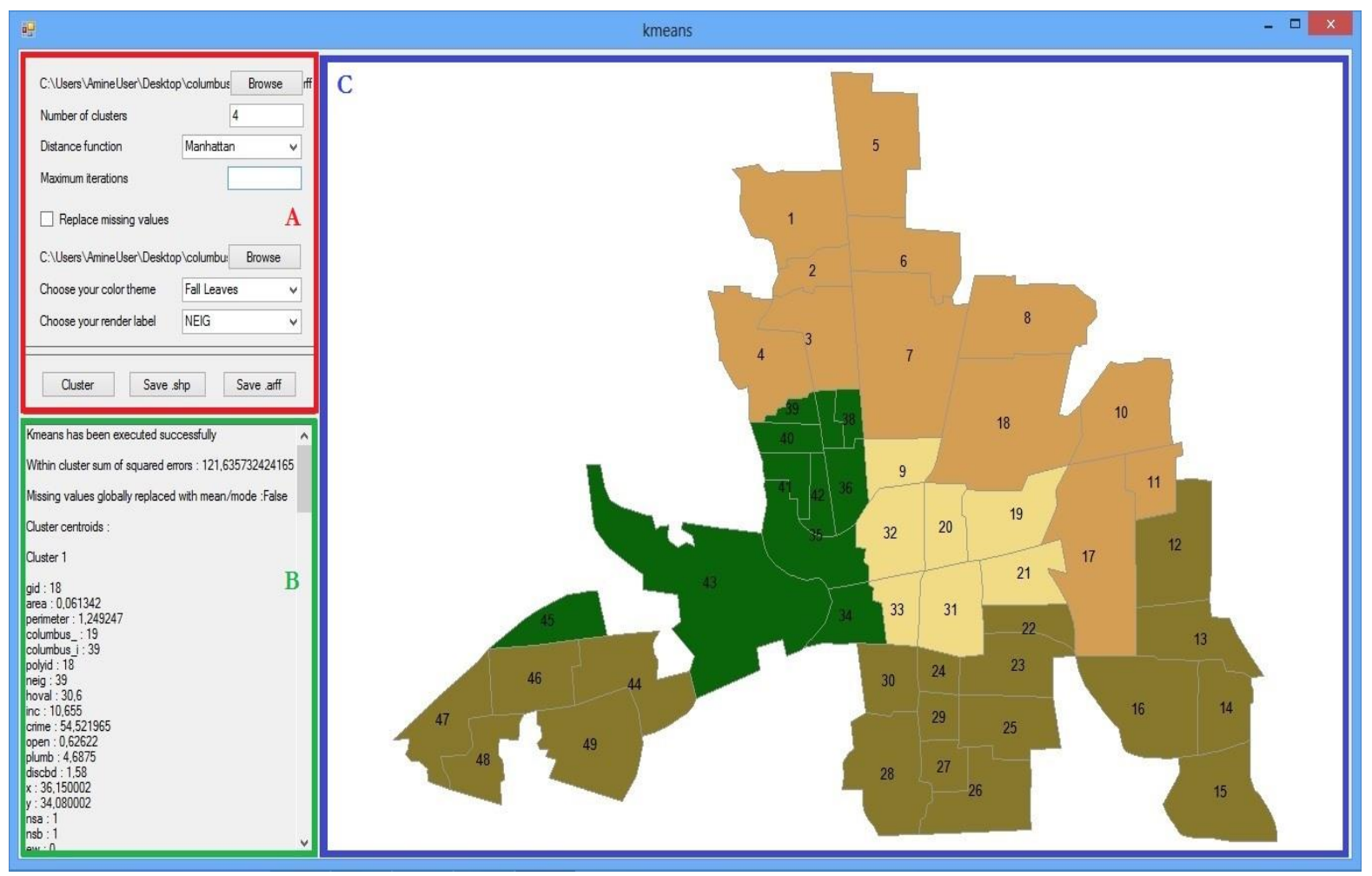

Figure 2: EasySDM Graphical User Interface and its three areas. Here, the K-means clustering algorithm has been applied $(\mathrm{k}=4)$. 


\subsection{Geographic pre-processing}

The geographic pre-processing is performed using the GDPM API. This step consists of extracting spatial relations and including them as new features to the data. These features represent the spatial objects as we used the instance granularity level. Each new feature will take as value, the existing spatial relation between the object represented by the new feature (columns) and the object represented by the corresponding instance (lines). If no spatial relation is found between the two objects, the corresponding cell takes the value 'no'. Two types of spatial pre-processing are available according to the types of the extracted spatial relationships:

\subsubsection{Distance relationships}

Three types of distance spatial relations have been considered: close, very close and far. The spatial relationship is chosen according to thresholds. Figure 3 presents the structure of the obtained arff file.

\begin{tabular}{|c|c|c|c|c|c|c|c|c|}
\hline & Att 1 & Att 2 & $\ldots$ & Att $\mathrm{m}$ & Obj 1 & Obj 2 & $\ldots$ & Obj n \\
\hline Obj 1 & & & & & Very close & no & & Close \\
\hline Obj 2 & & & & & no & Very close & & no \\
\hline Obj 3 & & & & & no & no & & no \\
\hline$\ldots$ & & & & & & & & \\
\hline Obj n & & & & & Close & no & & Very close \\
\hline
\end{tabular}

Figure 3: Structure of the arff file after the extraction of distance relationships.

\subsubsection{Topological relationships}

Eight types of topological spatial relationships have been considered: equal, disjoint, touches, within, overlaps, crosses, contains and covers. Figure 4 presents the structure of the obtained arff file.

\begin{tabular}{|c|c|c|c|c|c|c|c|c|}
\hline & Att 1 & Att 2 & $\ldots$ & Att $\mathrm{m}$ & Obj 1 & Obj 2 & $\ldots$ & Obj n \\
\hline Obj 1 & & & & & Touches & no & & Contains \\
\hline Obj 2 & & & & & no & Touches & & Touches \\
\hline Obj 3 & & & & & no & no & & no \\
\hline$\cdots$ & & & & & & & & \\
\hline Obj n & & & & & Contains & no & & Touches \\
\hline
\end{tabular}

Figure 4: Structure of the arff file after the extraction of topological relationships.

Once the geographical pre-processing step has been applied, the data can be used with classical DM algorithms. In this first version, the following representative algorithms have been integrated using the Weka software:
- Clustering by partitioning: K-means, Farthest First and Expectation Maximization.

- Density based clustering: Cobweb and DBscan.

- Classification: J48 and Naïve Bayes.

\subsection{Dynamic SDM algorithms}

Two naive SDM algorithms that can be applied directly on the spatial data have been implemented. The goal here is to illustrate the dynamic processing approach by simple examples.

\subsubsection{Regionalization}

While classical clustering methods do not guarantee that objects in the same cluster are contiguous, regionalization groups similar objects in contiguous regions. Figure 5 presents the difference between clustering and regionalization. Our implemented regionalization algorithm is divided into two steps. First, a classical clustering algorithm is applied (here we applied the FarthestFirst algorithm). Then, the obtained clusters are organized into contiguous regions. If non-contiguous objects exist in each cluster, they are separated in order to form a new cluster. This process is repeated until all regions become contiguous.

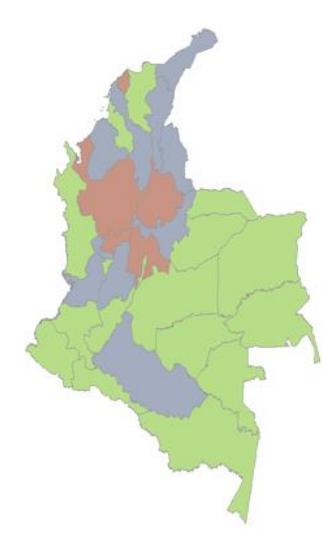

Clustering

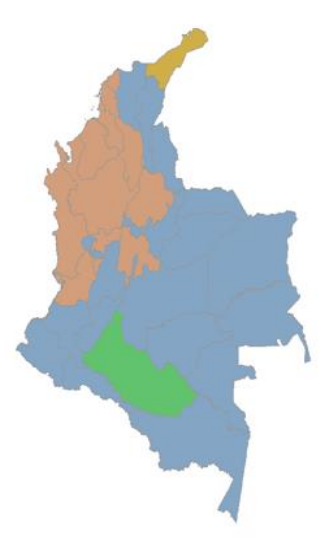

Regionalization
Figure 5: Difference between clustering and regionalization.

The algorithm is presented below:

Input: Dataset: D

Minimal number of regions: $k$.

Output: The assignments of elements to different regions. 


\section{Begin}

1. Apply Farthest First (D, k)

2. For each cluster $\mathrm{c}$ not yet checked, we create a region $r$ containing the first object of $\mathrm{c}$

2.1. For all other objects o from c:If (o touches at least one object of $r$ ): $r=r$ union $\{o\}$

2.2. All remaining objects (if any) are affected to a new cluster.

End

\subsubsection{Spatial Apriori}

Spatial association rule mining extracts rules in the form: $\mathrm{X}->\mathrm{Y}$, where $\mathrm{X}$ and $\mathrm{Y}$ are spatial predicates (intersection, contains, overlaps, disjointed, crosses, covers, covered and touches). We implemented a spatial variation of the Apriori algorithm that we call Spatial Apriori. It outputs the rule that has the highest confidence and the geographical objects that participate in this rule. Hence, Spatial Apriori can extract the best association rule found in a shape file with considered minimum support and minimum confidence. The different steps of Spatial Apriori algorithm are detailed below:

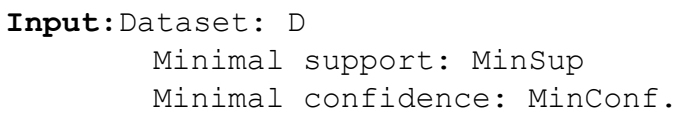

Begin

1. Predicates <- Apply all the families of spatial relations on all the elements of $D$.

2. Predicates <- Select only those for which the support is greater than Minsup.

3. Predicates <- Select the best predicate for each family.

4. Rules <- Combine the predicates and generate all possible association rules.

5. Rules <- select rules with confidence greater than MinConf.

6. Return the association rule with maximal confidence.

\subsection{Licence}

EasySDM is distributed under the GPL licence in order to guarantee free access to the platform, the source code and to any software build on it. Weka, GDPM and IKVM are already under the GPL licence. However, MapWinGis has been distributed under the MPL 1.1 licence, which is incompatible with GPL. Therefore, we contacted MapWinGis owners and they gave us authorization to use their API along with GPL licences we are using.

\section{EXPERIMENTS}

In order to test the functionalities of our new SDM platform, we conducted experiments using publically available spatial data on the GeoDa website (www.geodacenter.asu.edu/sdata). The main goal here is to illustrate the visualisation capabilities of EasySDM on a real benchmark.

\subsection{Description of the benchmark}

It contains Malaria incidence and population in Colombia. We selected information corresponding to the year 1998. The obtained benchmark contained 33 spatial objects (polygons) representing the 33 Colombia departments. These 33 departments were described only with 5 features: department name, department code, number of malaria incidences (MALARI98), total population (TP1998), rural population (RP1998) and urban population (UP1998). Geographical pre-processing added 33 new features to the data. Due to the nature of this benchmark (polygons) topological relations have been considered.

\subsubsection{Clustering}

We first conduct a clustering experiment using Kmeans $(\mathrm{k}=3)$ on the geographically pre-processed data. In this experiment, the department "San Andres" has been removed in order to be used later in the classification. As shown in Figure 6, three clusters have been created:

- The red cluster contains many departments especially from the center. Therefore, they contains many common borders. The number of Malaria incidences may be very high.

- The orange cluster contains departments from the North West of the country. The number of Malaria incidences is high as well as the population, which is more urban than rural. 
- The yellow cluster groups departments which has less common borders, less population and less Malaria cases. The population is more rural than urban.

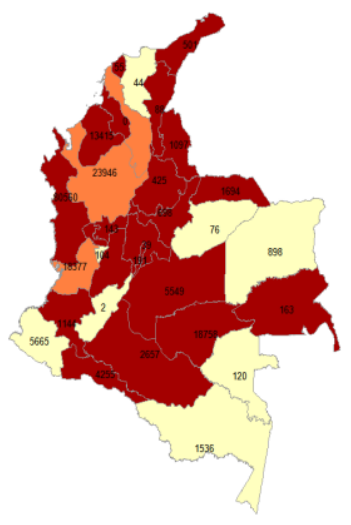

MALARI98
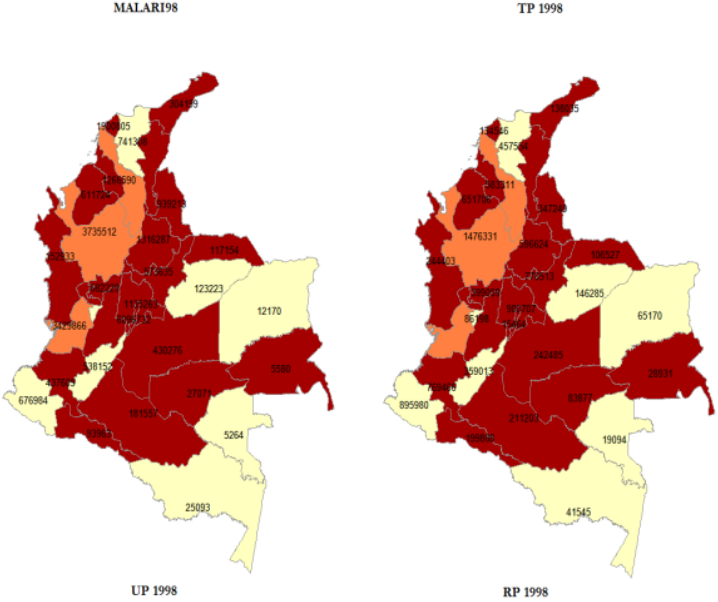

Figure 6: Clustering using K-means $(\mathrm{k}=3)$ on the first benchmark. The number of Malaria incidence (MALARI98) and the rural (RP 1998), urban (UP 1998) and total population (TP 1998) are presented for each department.

\subsubsection{Classification}

We used the 32 already classified departments to build a Naive Bayes classifier which was used to classify the left "San Andres" department (composed of two islands). As shown in Figure 7, "San Andres" has been affected to the yellow cluster since it has no Malaria incidence $(0)$, a small population (69525) and no common border with any other department.

\subsubsection{Association rules}

Finally, we apply the naive variation of Apriori to this benchmark. The extracted rule is the following (support and confidence have been set to 0 ):

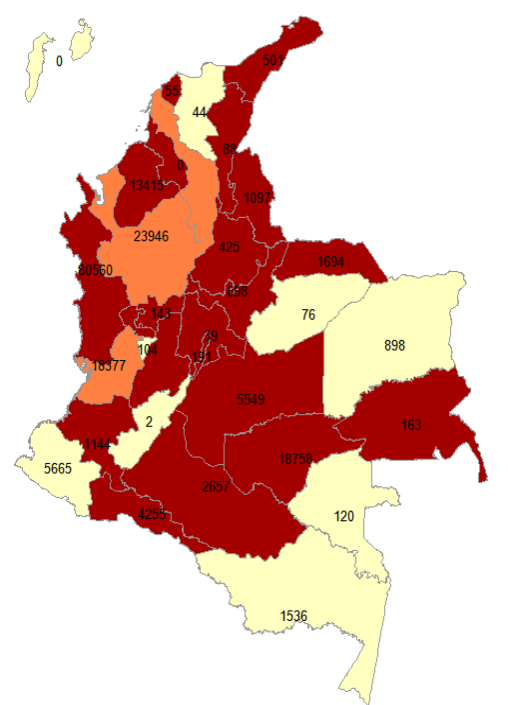

Figure 7: Classification of the "San Andres" department (the two islands) using a Naïve Bayes classifier. The number of Malaria incidences is indicated for each department.

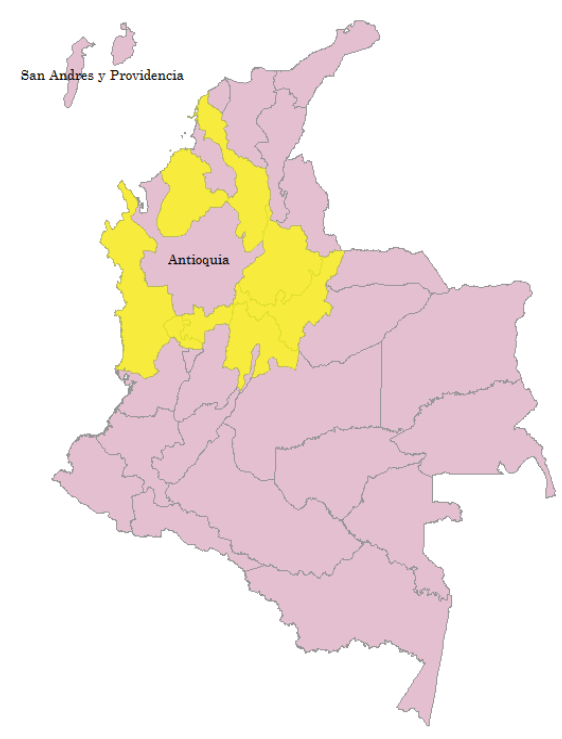

Figure 8: The departments verifying the obtained rule by Spatial Apriori

\section{Intersects $($ Antioquia $)=>$ Disjoint $($ San Andres $)$}

This rule has a value of 0.24 in terms of support and 1 in terms of confidence. The departments verifying this rule are presented in yellow in Figure 8. The obtained rule is obvious and may not be valuable for the user. Since the purpose of the platform is to explain SDM to novice users we did not remove obvious rules. This can be included in future versions. 


\section{CONCLUSION}

In this work, we propose an open source and easy to use SDM platform named EasySDM. It integrates classical DM methods implemented in the Weka platform after a Geographical pre-processing step. Moreover, it contains two naïve algorithms that consider the spatial relations inside the mining process without any pre-processing. The main contribution concerns the integration of an internal geographic visualization of the spatial data before and after applying the algorithms. EasySDM can also be interfaced with any GIS to offer external visualization and to take advantage of GIS functionalities. Therefore, we believe that it can be used in the academic area. The main expected improvements of EasySDM consist of enriching it with existing algorithms from the literature.

\section{REFERENCES}

Anselin, L., Syabri, I., Kho, Y., 2006. GeoDa: An Introduction to Spatial Data Analysis. Geographical Analysis 38, 5-22.

Appice, A., Lanza, A., Malerba, D., 2007. An Integrated Platform for Spatial Data Mining Within a GIS Environment, in: ICDE Workshop on Spatio-Temporal Data Mining, pages 507-516. IEEE Computer Society.

Bogorny, V., Palma, A.T., Engel, P., Alvares, L.O., 2006. Weka-gdpm: Integrating classical data mining toolkit to geographic information systems, in: SBBD Workshop on Data Mining Algorithms and Aplications, Florianopolis, Brasil, pp. 1620.

Goebel, M., Gruenwald, L., 1999. A Survey of Data Mining and Knowledge Discovery Software Tools. SIGKDD Explor. Newsl. 1, 20-33.

Guo, D., 2008. Regionalization with Dynamically Constrained Agglomerative Clustering and Partitioning (REDCAP). Int. J. Geogr. Inf. Sci. 22, 801-823.

Guo, D.,Mennis. J. 2009.Spatial data mining and geographic knowledge discovery-An introduction. Computers, Environment and Urban Systems. 33. 403-408.

Hall, M., Frank, E., Holmes, G., Pfahringer, B., Reutemann, P., Witten, I.H., 2009. The
WEKA Data Mining Software: An Update. SIGKDD Explor. Newsl. 11, 10-18.

Han, J., Koperski, K., Stefanovic, N., 1997. GeoMiner: A System Prototype for Spatial Data Mining, in: Proceedings of the 1997 ACM SIGMOD International Conference on Management of Data, ACM, New York, NY, USA, pp. 553-556.

Jiawei Han, Y.F., 1996. DBMiner: A System for Mining Knowledge in Large Relational Databases. KDD-96 Proceedings, 250-255.

Elder .J. F., Abbott. D.W, 1998. A Comparison of Leading Data Mining Tools. Presented at the Fourth International Conference on Knowledge Discovery and Data Mining, New York.

Lazarevic, A., Fiez, T., Obradovic, Z., 2000. A software system for spatial data analysis and modeling, in: Proceedings of the 33rd Annual Hawaii International Conference on System Sciences.

Levine, N., others, 2004. CrimeStat III: a spatial statistics program for the analysis of crime incident locations (version 3.0). Houston (TX): Ned Levine \& Associates/Washington, DC: National Institute of Justice.

May, M., Savinov, R., 2001. An Architecture for the SPIN! Spatial Data Mining Platform, in: Proc. New Techniques and Technologies for Statistics. pp. 467-472.

Miller, H.J., 2004. Tobler's First Law and Spatial Analysis. Annals of the Association of American Geographers 94, 284-289.

Ouattara, M., 2010. Fouille de données: vers une nouvelle approche intégrant de façon cohérente et transparente la composante spatiale. Université Laval.

Rinzivillo.S, Turini. F,Bogorny. V, Körner. C, Kuijpers. B, andMay,. M.2008. Knowledge discovery from geographical data. Mobility, Data Mining and Privacy, pp. 243-265,

Witten, I.H., Frank, E., 2005. Data Mining: Practical Machine Learning Tools and Techniques, Second Edition. ed. Morgan Kaufmann Publishers Inc., San Francisco, CA, USA. 